\title{
In favour of the time variable in classical thermodynamics
}

\author{
PierGianLuca Porta Mana \\ Perimeter Institute for Theoretical Physics, Canada \\ $<$ Imana@perimeterinstitute.ca>
}

19 January 2011

(first drafted 29 October 2010)

\begin{abstract}
A case for the teaching of classical thermodynamics with an explicit time variable, with phenomena involving changes in time, is made by presenting and solving a exercise in textbook style, and pointing out that a solution accords with experiment. The exercise requires an explicit treatment of the time variable. Further arguments are given for the advantages of an explicit time variable in classical thermodynamics, and against some standard terminology in this theory.

PACS numbers: 05.70.-a,64.10.+h,01.40.gb

MSC numbers: 80-01,97M50,34-04
\end{abstract}

to Laura

'i mean if you dont change in 2 years or even in a day, then whats the point?'

L. Pasichnyk

\section{Introduction}

The way I learnt classical thermodynamics in my undergraduate studies was very different from the way I learnt mechanics and electromagnetism. The most important part of these two disciplines was about changes in time and the laws governing these changes; in fact, 
their fundamental laws are differential equations involving time derivatives. In thermodynamics, instead, there was no change; all exercises were mainly about static equilibrium situations and their comparison: the fundamental laws of thermodynamics did not involve time and were stated by means of differentials, not derivatives.

This difference in philosophy and mathematics was frustrating. First of all, I had a feeling of a lack of unity in Physics. For mechanics and electromagnetism could easily be made to interact with each other, proving to be only two simplified aspects of that variety of physical phenomena that every day fills our senses with marvel. Yet, our senses also tell us that this variety includes thermal phenomena; but this was not reflected in classical thermodynamics: its interaction with the other two disciplines was scarce and limited to trivial problems.

Time and change were not completely absent, of course. 'Quasistatic' processes were named (with a lot of verbal exorcisms owing to their 'non-reality'), but never developed mathematically with the introduction of a time variable. Time only appeared explicitly in very elementary problems of heat conduction that involved heat and temperature only, or of diffusion.

I believe my experience is common to many students, especially in departments of physics, as can be evinced by a look at thermodynamic textbooks. For example, in $\S 2.1$, p. 30, of the widely used book by Zemansky [1] we read:

Thermodynamics does not attempt to deal with any problem involving the rate at which a process takes place. The investigation of problems involving the time dependence of changes of state is carried out in other branches of science, as in the kinetic theory of gases, hydrodynamics, and chemical kinetics.

Or in Wilson's [2, § 1.1, p. 2]:

Another way in which thermodynamics differs from other subjects is that it only deals with equilibrium states and with transitions from one equilibrium state to another.

Callen [3, § 1-1, p. 6] says, more concisely but in italics:

thermodynamics describes only static states of macroscopic systems. 
There are exceptions. There are textbooks in which the fundamental principles of thermodynamics are explained and expressed from the beginning in terms of change and time; for example Sandler's [4, see chs 3, 4], Moran \& Shapiro's [5, see chs 2, 6], and Bohren \& Albrecht's refreshing textbook [6] (see also Winterbone's [7, passim]). One can also find an occasional article, like Murphy's [8], where thermodynamic variables are treated with an explicit time dependence without reference to kinetic theory or 'irreversible thermodynamics' (I do not count exercises about heat conduction as they typically involve heat and temperature only). So there are courses in which thermodynamics is taught that way; such courses seem to be more numerous in departments of engineering and chemistry than of physics.

Why is thermodynamics taught without the time variable? and in which kind of courses and departments is it taught differently? It is not my purpose here to answer these questions; for the first, you may take a look at the researches by Truesdell et al. [9-12]. Rather, I should like to show, to those students and teachers who have had experiences similar to mine, that thermodynamics can be approached in a way more similar to mechanics and electromagnetism, with change and time. Cases for the explicit appearance of time in thermodynamics were already made by Eckart [13]; by Bridgman [14, see e.g. pp. 24-26]; extensively and intensively by Menger [15]; very forcefully, with telling historical, physical, and mathematical arguments, by Truesdell and his school of 'rational thermodynamics' (see e.g. [9-11; 16-24]); and by Bohren \& Albrecht [6, §§ 1.8, 3.1]. Here I will show this time-dependent thermodynamics in action by proposing and solving a thermodynamic exercise in a typical textbook style. Note that the solution of the exercise can be and is experimentally verified in a simple undergraduate laboratory experiment, as will be discussed later in \$3, so there is no arguing that the exercise has no physical meaning or application. The exercise is suited to students with a knowledge of differential equations, but could be re-expressed in a simpler way that avoids direct mention of differential equations (as is done with the harmonic oscillator, e.g., in basic courses).

Afterwards I will argue that the presentation of thermodynamics with an explicit time dependence and time derivatives is more advantageous, more general, and physically, mathematically, philosophically more appealing that the one based on the absence of time and the mathematics of differentials. 


\subsection{Classical thermodynamics with time}

Before presenting the exercise, let us see how the time variable is introduced in classical thermodynamics. The change is very simple:

- The variables describing the system depend on time: the independent ones directly, and the dependent ones indirectly through their dependence on the independent variables. For example, if volume $V$ and temperature $T$ are the independent variables, and pressure $p$, internal energy $U$, entropy $S$ the dependent ones, we shall have $V(t), T(t), p[V(t), T(t)], U[V(t), T(t)]$, etc.

- The differentials usually appearing in the first and second laws are recognized to be differentials with respect to time, and therefore replaced by time derivatives. So the first and second law assume the forms

$$
\dot{U}=\Phi-P, \quad \dot{S} \geqslant \frac{\Phi}{T} .
$$

In these equations, $\dot{U}:=\mathrm{d} U / \mathrm{d} t$ and $\dot{S}:=\mathrm{d} S / \mathrm{d} t$ are the rates of change of internal energy and entropy, $\Phi$ is the heating, or rate at which the system is heated, and $P$ is the working, or rate at which the system does work. The latter is usually specified in terms of the independent and dependent variables of the system and their rates of change, e.g. $P=p \dot{V}$. (The term 'power' is usually reserved for the sum of the working and the rate of change of kinetic energy, the latter being often negligible in thermodynamics.)

This formulation was discussed by Menger [15] and is presented in Truesdell [18, Lecture 26; 19; 9, Lecture 1 and Appendix1A], Truesdell \& Bharatha [10], Owen [20], Samohýl [21, ch. II], and the already mentioned Sandler [4]. See also Müller [25, § 5.1].

Now to our exercise:

\section{A textbook exercise in thermo-dynamics}

A vessel communicating with a tube as in the set-up of fig. 1 is filled with a mass $M$ of an ideal gas. This set-up can be idealized as a tube of constant cross-section area $A$ as in fig. 2. the gas is enclosed between the closed base of the tube and a piston of mass $m$ that fits the tube and can move along it without friction. The variable distance of the piston from the base is denoted by $h(t)$. A constant force $f$ acts on the piston from the outside (the force can e.g. consist 


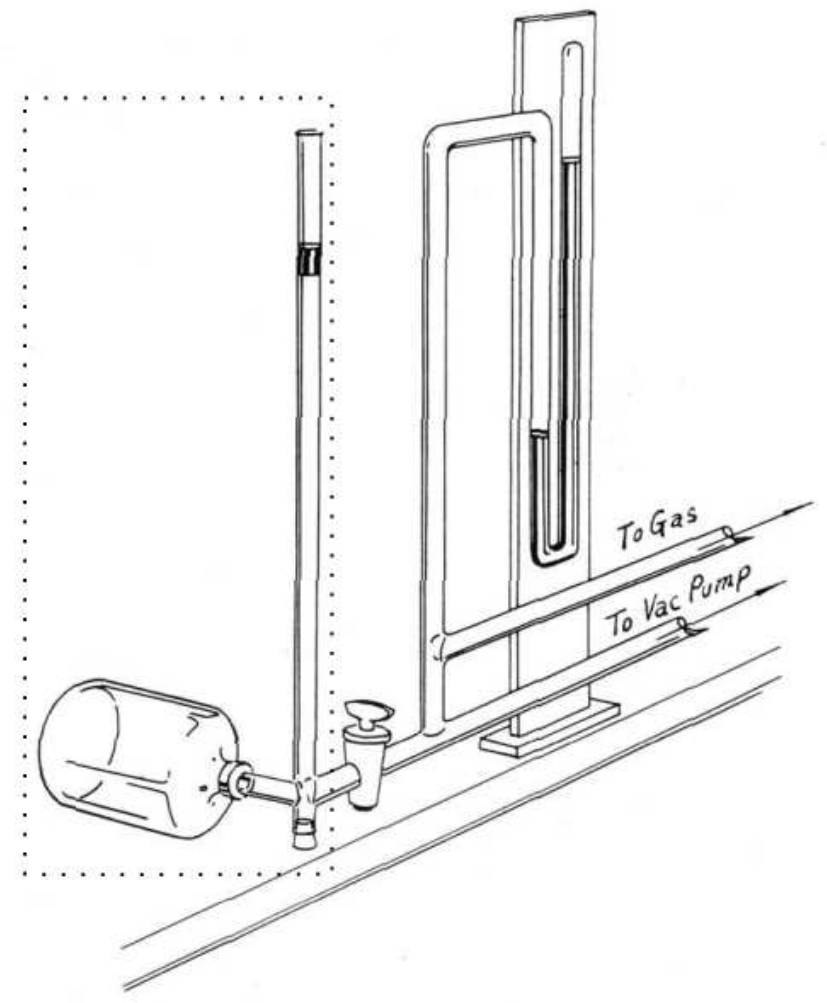

Figure 1: Assmann-Rüchardt-Schufle apparatus. The part of the apparatus inside the dotted rectangle is idealized in fig. 2. notice the piston in the left tube. With this apparatus the solution of problem (2), shown in fig. 4, can be experimentally observed. (Reprinted and adapted from Schufle [26] with the permission of the American Chemical Society)

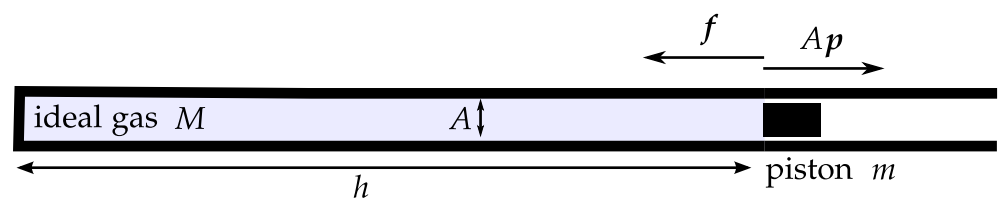

Figure 2: Idealization of the experimental set-up of fig. 1 
in atmospheric pressure, and also in the weight of the piston itself if the tube is in a vertical position). The gas is homogeneous at all times, and is characterized by an individual massic gas constant $R$ (i.e. $R=\bar{R} / M_{\mathrm{m}}$ with $\bar{R}$ the ideal-gas constant and $M_{\mathrm{m}}$ the molar mass of the gas) and a linear relation between internal energy and temperature with massic heat capacity $c$. Consider the following two distinct problems:

A. Until time $t_{0}$ the piston is at a distance $L$ from the base of the tube and the gas is in equilibrium. Starting at time $t_{0}$ heating is suddenly provided thereafter at a rate $\Phi(t)$. How must this heating rate depend on time if the distance of the piston from its initial position has to change like

$$
h(t)=L+\lambda \frac{\left(t-t_{0}\right)^{3}}{\tau^{3}}
$$

with $\lambda$ and $\tau$ two constant positive parameters? How do pressure and temperature change?

B. At time $t_{0}$ the piston is at a distance $L$ from the base of the tube, with nought velocity but an initial negative (i.e., towards the base) acceleration $a$. The gas is adiabatically insulated at all times, i.e. the heating vanishes, $\Phi(t)=0$. What is the motion of the piston after time $t_{0}$ ? If necessary, solve the problem using numerical methods with the values provided below.

For both problems, plot the time dependence of heating, piston position, pressure, and temperature assuming

$$
\begin{aligned}
L & =20 \mathrm{~m}, \\
A & =10^{-4} \mathrm{~m}^{2}, \\
M & =2 \times 10^{-3} \mathrm{~kg}, \\
f & =9 \mathrm{~N}, \quad \text { corresponding to a pressure } f / A=9 \times 10^{4} \mathrm{~Pa}, \\
m & =10^{-2} \mathrm{~kg}, \\
R & =3 \times 10^{2} \mathrm{~J} /(\mathrm{kg} \cdot \mathrm{K}), \\
c & =7 \times 10^{2} \mathrm{~J} /(\mathrm{kg} \cdot \mathrm{K}), \\
\lambda & =5 \times 10^{-1} \mathrm{~m}, \\
\tau & =3 \mathrm{~s}, \\
a & =-10 \mathrm{~m} / \mathrm{s}^{2} \quad(\text { as in free fall). }
\end{aligned}
$$

(If the value of $L$ appears impractically large, consider that this is an idealization of the real set-up of fig. 1 the distance $L$ of the exercise 
is an effective distance given by $L=L_{\mathrm{r}}+V_{\mathrm{v}} / A$, where $L_{\mathrm{r}}$ is the distance of the piston from the base of the real tube and $V_{\mathrm{v}}$ is the volume of the vessel connected to the latter; the same holds for $h$. The real tube can be between one and two metres long.)

\section{Solution and remarks}

Let us denote the gas' pressure, temperature, and internal energy by $p(t), T(t), U(t)$. The equations relevant to the problem are:

- Momentum balance for the piston, upon which act the pressure of the gas $p(t)$ and the external force $f$ :

$$
A p(t)-f-m \ddot{h}(t)=0,
$$

where a dot stands for the time derivative: $\dot{h}:=\mathrm{d} h / \mathrm{d} t$, etc.

- Energy balance for the gas:

$$
\dot{U}(t)=\Phi(t)-p(t) A \dot{h}(t),
$$

where $p(t) A \dot{h}(t)$ is the working done by the gas, since the volume is obviously given by $A h(t)$ and $A$ is constant. Note that $\Phi(t)=\dot{Q}(t)$, where $Q(t)$ is the integrated heating from some arbitrarily specified time until time $t$.

- Constitutive law for the pressure of an ideal gas:

$$
p(t)=\frac{M R T(t)}{A h(t)} .
$$

And finally

- constitutive equation for the gas' internal energy, as specified in the problem:

$$
U(t)=\operatorname{McT}(t) .
$$

The equations above constitute a system of coupled ordinary differential equations for the unknown $h, T, p, U$, given $\Phi(t)$. The system can be easily simplified. Substituting the expression for the pressure (6) in the momentum balance (4) we can express $T$ in terms of $h$ :

$$
T(t)=\frac{1}{M R} h(t)[f+m \ddot{h}(t)]
$$

from which, substituting in (6) and (7), we also obtain an expression for the pressure:

$$
p(t)=\frac{1}{A}[f+m \ddot{h}(t)],
$$


and the internal energy:

$$
U(t)=\frac{c}{R} h(t)[f+m \ddot{h}(t)] .
$$

From the latter two equations and the energy balance (5) we finally obtain an expression relating $h$ and $\Phi$ :

$$
\Phi(t)=\left(1+\frac{c}{R}\right) \dot{h}(t)[f+m \ddot{h}(t)]+\frac{c m}{R} h(t) \dddot{h}(t) .
$$

If the position function $h$ is given, the equation yields the heating $\Phi$; if the heating is given, this is an ordinary differential equation of third order from which $h$ can be obtained, possibly with numerical methods, given appropriate initial conditions for $h, \dot{h}$, and $\ddot{h}$.

Problem $\mathrm{A}$ is easily solved by substituting the expression (2) for $h$ into (9). We find

$$
\Phi(t)=\frac{6 c L \lambda m}{R \tau^{3}}+\frac{3 f \lambda(c+R)}{R \tau^{3}}\left(t-t_{0}\right)^{2}+\frac{6 m \lambda^{2}(4 c+3 R)}{R \tau^{6}}\left(t-t_{0}\right)^{3} .
$$

Using equations $(8 \mathrm{a})$ and $(8 \mathrm{~b})$ we also find the time dependence of pressure and temperature:

$$
\begin{aligned}
& p(t)=\frac{f}{A}+\frac{6 \lambda m}{A \tau^{3}}\left(t-t_{0}\right) \\
& T(t)=\frac{f L}{M R}+\frac{6 \lambda L m}{M R \tau^{3}}\left(t-t_{0}\right)+\frac{f \lambda}{M R \tau^{3}}\left(t-t_{0}\right)^{3}+ \\
& \frac{6 \lambda^{2} m}{M R \tau^{6}}\left(t-t_{0}\right)^{4}
\end{aligned}
$$

These solutions are shown in fig. 3 for the numerical values (3). With those values, $\Phi$ is dominated by its quadratic term in time, $T$ by its constant and cubic terms.

Now to problem $\mathrm{B}$. Since the process is adiabatic, $\Phi(t)=0$, the differential equation (9) can be rewritten as

$$
\left(1+\frac{c}{R}\right) \dot{h}(t)[f+m \ddot{h}(t)]+\frac{c m}{R} h(t) \dddot{h}(t)=0,
$$

with the initial conditions

$$
h\left(t_{0}\right)=L, \quad \dot{h}\left(t_{0}\right)=0, \quad \ddot{h}\left(t_{0}\right)=a .
$$


The differential equation above can be integrated once by rewriting it as

$$
\frac{m \dddot{h}(t)}{f+m \ddot{h}(t)}=-\left(1+\frac{R}{c}\right) \frac{\dot{h}(t)}{h(t)}
$$

and noting that each side is the derivative of a logarithm. Integrating between $t_{0}$ and $t$, exponentiating the logarithms, rearranging, and using the initial conditions (14) we obtain

$$
[f+m \ddot{h}(t)] h(t)^{\gamma}=[f+m a] L^{\gamma}
$$

with $\gamma:=1+R / c$, the ratio of specific heats of the gas. Comparison with expression (8b) for the pressure shows that this equation is the law for adiabatic processes, $p(t)[A h(t)]^{\gamma}=p\left(t_{0}\right)\left[A h\left(t_{0}\right)\right]^{\gamma}$.

This differential equation does not seem to be solvable analytically, so we proceed by numerical integration using the values (3). Note that these values could correspond to having the tube placed vertically and suddenly dropping the piston in it at time $t_{0}$.

The numerical solution is shown in fig. 4. We observe an oscillatory behaviour of all quantities, with obviously the same period of circa $0.8 \mathrm{~s}$. The oscillation amplitude of the piston is around $1.5 \mathrm{dm}$, that of the pressure around $1 \mathrm{kPa}$, and that of the temperature around $1 \mathrm{~K}$. One can show numerically that changing the initial acceleration only changes the amplitudes of the oscillations but not their period.

Now let us see whether the differential equation (9) admits any approximate form that can be solved analytically. If the inertial term $m \ddot{h}(t)$ is small in comparison to the force $f$ and the variations of $h(t)$ are small in comparison to its initial value $L$, i.e.

$$
\frac{m \ddot{h}(t)}{f} \text { and } \frac{h(t)-L}{L} \text { small, }
$$

then (9) can be approximated by

$$
\Phi(t)=\left(1+\frac{c}{R}\right) f \dot{h}(t)+\frac{c m L}{R} \dddot{h}(t) .
$$

Integrating between $t_{0}$ and $t$ and defining

$$
\bar{h}(t):=h(t)-h\left(t_{0}\right)-\frac{c m L}{c+R} \ddot{h}\left(t_{0}\right), \quad Q(t):=\int_{t_{0}}^{t} \Phi(s) \mathrm{d} s
$$


we obtain

$$
Q(t)=\left(1+\frac{c}{R}\right) f \bar{h}(t)+\frac{c m L}{R} \ddot{\bar{h}}(t) .
$$

This equation describes a driven harmonic motion and can be solved exactly in terms of integrals of $Q$. The intrinsic period is

$$
\tau_{\mathrm{o}}=2 \pi \sqrt{\frac{c m L}{(c+R) f}}=0.78 \mathrm{~s},
$$

which is approximately equal to that found numerically in the solution to problem $B$. In fact, that solution does reasonably satisfy the conditions (17) and one can compare the numerical result with those obtained using eq. (19b). For $t_{0} \leqslant t \leqslant \tau$ it is found that the difference in the prediction of $h(t)$ does not exceed $10^{-3} \mathrm{~L}$.

The solution found in problem $B$ can be experimentally observed with an Assmann-Rüchardt apparatus [27; 28] as modified by Schufle [26], which is exactly our real set-up of fig. 1 With this apparatus, the oscillatory behaviour we found is used to calculate the ratio of specific heats of the gas, which in our case is given by $\gamma=1+R / c$, by measuring the period $\tau_{\mathrm{o}}$ approximately given by eq. (20). The values (3) are indeed approximately taken from Schufle's article, and our numerical solution gives with good approximation the period of oscillation observed in his experiments.

It should be remarked however that for a smaller volume of gas, i.e. smaller $L$, or bigger piston mass $m$, the approximations (17) do not hold and the approximate equation (18) gives results that differ appreciably from those of eq. (9). Assume e.g. the values

$$
\begin{aligned}
L & =1 \mathrm{~m}, \\
A & =10^{-4} \mathrm{~m}^{2}, \\
M & =2 \times 10^{-3} \mathrm{~kg}, \\
f & =19 \mathrm{~N}, \\
m & =1 \mathrm{~kg}, \\
R & =3 \times 10^{2} \mathrm{~J} /(\mathrm{kg} \cdot \mathrm{K}), \\
c & =7 \times 10^{2} \mathrm{~J} /(\mathrm{kg} \cdot \mathrm{K}), \\
a & =-10 \mathrm{~m} / \mathrm{s}^{2},
\end{aligned}
$$

which represent a vertical $1 \mathrm{~m}$ tube with a piston of mass $1 \mathrm{~kg}$ acted upon by atmospheric pressure. With these values eqs (9) and (18) 

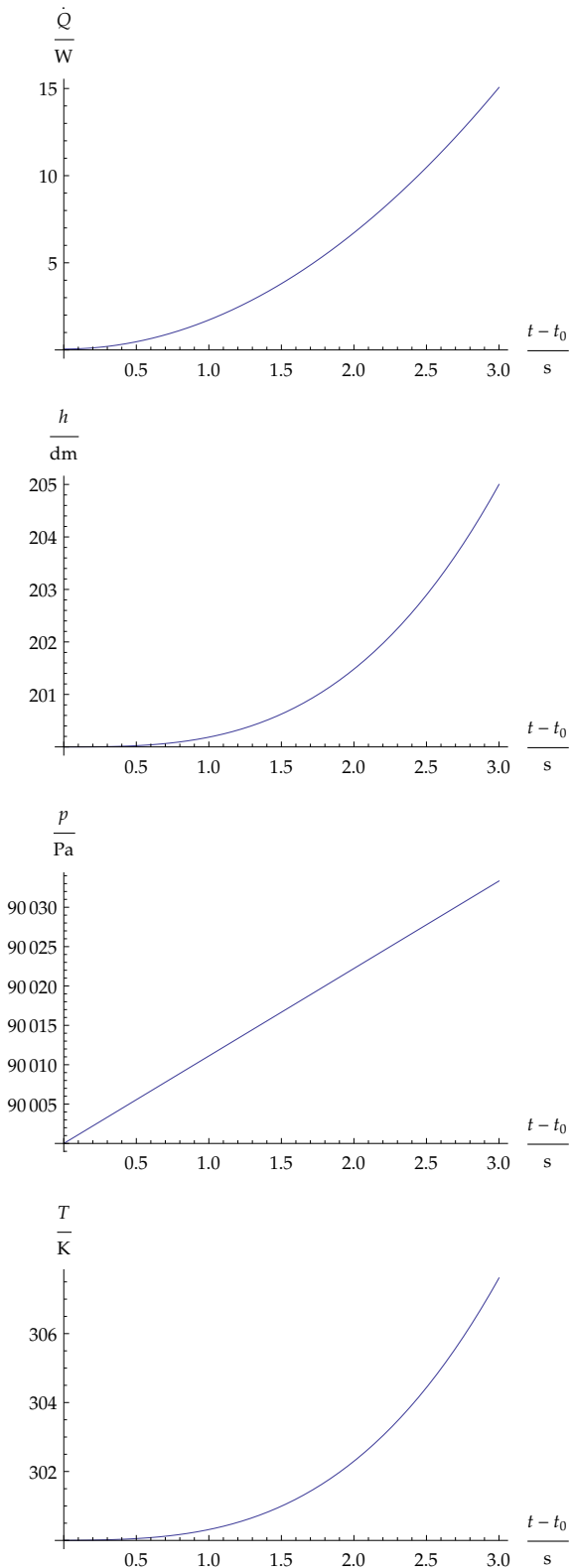

Figure 3: Solution for problem $\mathrm{A}(t)=L+\lambda \frac{\left(t-t_{0}\right)^{3}}{27 \tau^{3}}$ and the numerical values (3) 

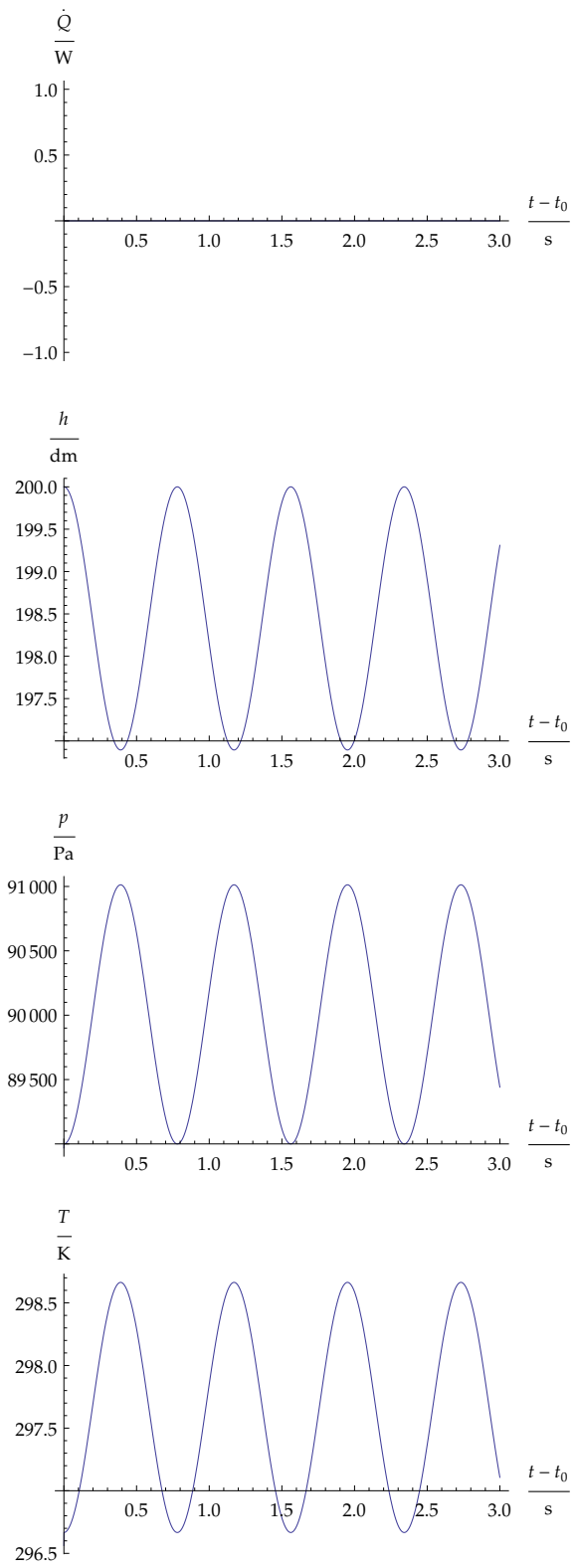

Figure 4: Solution for problem B $\Phi(t)=0 \mathrm{~W}$, an initial negative acceleration of $\ddot{h}\left(t_{0}\right)=a$, and the numerical values (3) 

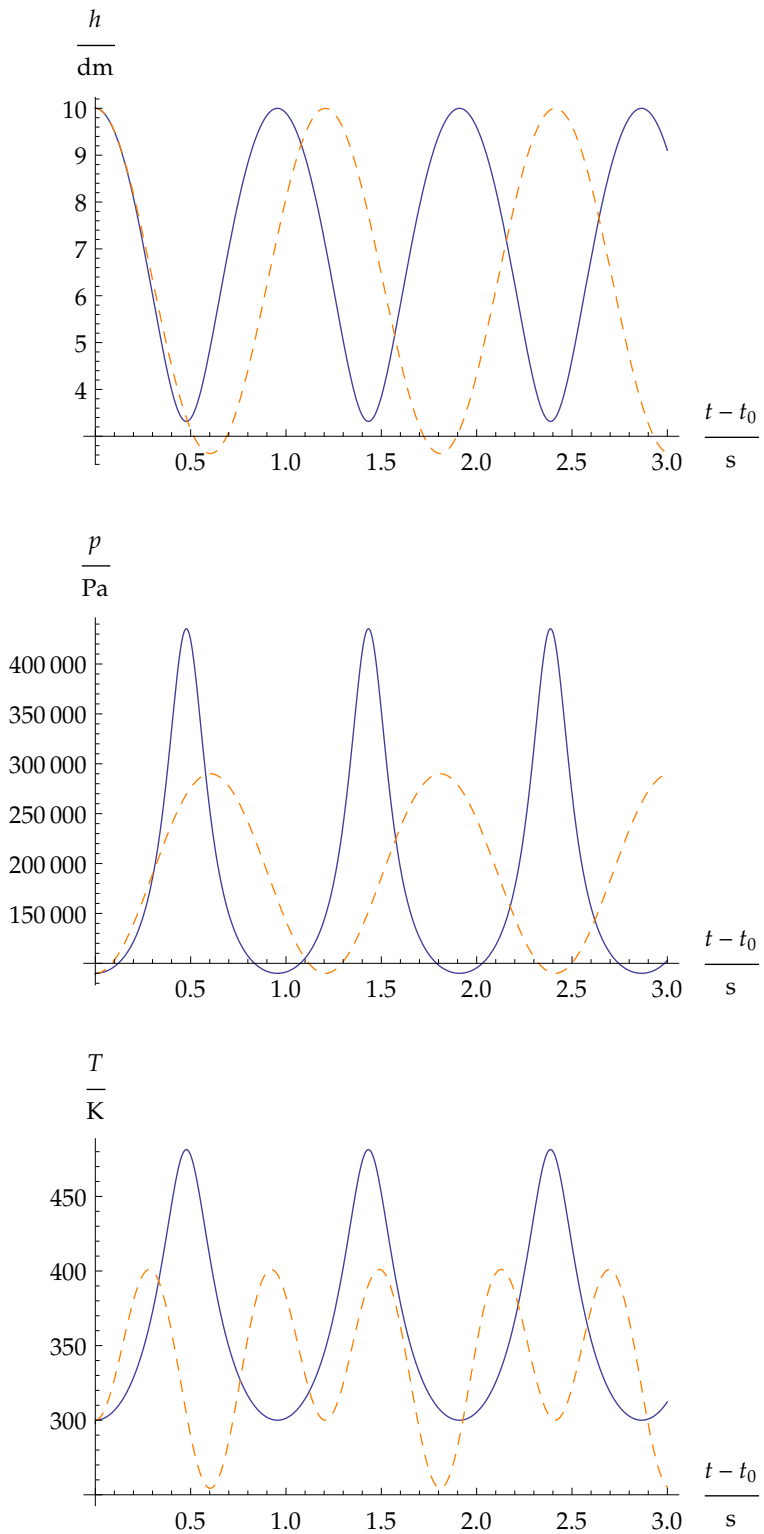

Figure 5: Comparison of solutions for problem $B$ with the numerical values (21), using the exact equation (9) (continuous blue line) and the approximate equation (19) (dashed orange line) 
give distinctly different solutions, as can be seen in fig.5. continuous blue line for eq. (9) and dashed orange line for eqs (18). Not only is the period of the piston's motion different: the first solution is an anharmornic oscillation; the second, harmonic. As expected, eq. (9) can present more interesting behaviours than just harmonic motion.

\section{Arguing for the time variable in classical thermodynamics}

\subsection{Advantages}

With the solution and analysis of our little textbook exercise I hope to have provided one more piece of evidence that time has its appropriate place in classical thermodynamics and thermodynamic courses. Its explicit presence has many advantages:

First, it makes thermodynamics feel closer in spirit to mechanics and electromagnetism, giving a more unified view of physics.

Second, it allow us to avoid any mention of differentials, especially 'inexact differentials', and therefore does not require notions from the mathematics of differential forms. Moreover, the fundamental laws (1), stated with respect to time, show why there cannot be differentials related to heating and working: separately, they are not the rates of change of anything. (For a beautiful, rigorous, and fruitful use of differential forms in physics see Burke [29; 30], Bossavit [31], Hehl \& Obukhov [32], van Dantzig [33; 34], Schouten [35], Truesdell \& Toupin [16, ch. F].)

Both points above allow, as a third advantage, an easier study of problems in which thermal, mechanical, and electromagnetic phenomena appear together. Think what would happen if the piston of our exercise had an electric charge and the tube was in a magnetic field. This kind of problems is often avoided because of the clash between the mathematics typically used in thermodynamics and in mechanics, a clash that leads to ugly mathematical gymnics. An example is given in Schufle's article [26]: he must derive the equation of a harmonic oscillator in a set-up similar to that of our exercise. He uses differentials in deriving the necessary relations between thermodynamic quantities, but then he must combine these with mechanical ones, arriving at an expression with the differential of a force. At that point he has to concede that the force is related to the double timederivative of position, revealing that all preceding differentials were 
but time derivatives in disguise. Our solution in $\S 3$ was obtained in a mathematically seamless way instead, owing to the presence of time in the basic thermodynamic equations.

Fourth - and this is a very important point not only for teachers and students, but also for researchers - it makes it possible to study more interesting thermodynamic systems for which the pressure or other quantities depend not only on volume and temperature but also on their rates of change, say $p(V, T, \dot{V})$, as it happens e.g. in synovial fluids [36; 37] or in pyroelectric materials [38]. The mathematics of differentials can hardly handle such systems. On this generalization I recommend the very lucid and simply written work of Samohýl [21], especially ch. II.

Fifth, it is full of physical and mathematical pedagogic potentials, as the interesting third-order ordinary differential equation (9) shows: it leads to a rich class of mathematical problems and physical behaviours (the driven harmonic oscillator being only a special case), providing also pedagogic material for laboratory classes.

\subsection{Common, weak objections}

In conversations with colleagues on this topic two main kinds of objection appear against a presentation of thermodynamics with an explicit presence of time. I find both very weak:

The first objection is that thermodynamics would only be concerned about equilibrium and relations between equilibria, and would have nothing to say about rates of change. I should rather call such a theory thermostatics; cf. Eckart [13]. But why should we limit ourselves to thermostatics, especially when experiment shows that the application to time-dependent phenomena is justified as we have seen e.g. in Schufle's experiment above? We do not limit ourselves to statics within mechanics. Some people have even the extreme view that there is no proper science of the rate of change of thermal systems: thermodynamics can only be thermostatics. I do not give much weight to this view because it is simply contrary to experience, experimental evidence, and practice. According to this view all theories about non-equilibrium thermodynamics, routinely taught and used by engineers and taught in some higher physics courses, would be nonsense. That is preposterous. (Some people even say temperature is defined only at equilibrium, contradicting themselves each time they check a weather thermometer.) 
The second objection is that an explicit time dependence and the appearance of differential equations is unwarranted for high-school and undergraduate students in their first years. But would not classical mechanics present exactly the same problem? Yet, many mechanical problems, like the harmonic oscillator or ballistic problems, can be and are routinely approached and solved without the need to study the full theory of differential equations. The same could surely be done with thermodynamics. Moreover, is using non-intuitive and typically poorly explained 'inexact differentials', without explicitly introducing the theory of differential forms, really easier than using rates of change?

\subsection{Inadequacy of the standard terminology of classical thermodynamics}

Part of the inertia against the time variable in thermodynamics comes from the terminology taught in thermodynamic courses. Indeed, even those who are keen on allowing the presence of time in classical thermodynamics do so only after some verbal exorcisms. They say, e.g., that a process is 'quasi-static' or that the thermodynamic system 'passes through a sequence of equilibrium states', or other similar phraseologies. I find most of these phraseologies misleading, counter-intuitive, and argue that we should change them. Their inadequacy can be seen from different points of view:

First: In mechanics, electromagnetism, quantum theory we use particular terminologies to indicate that specific equations and laws apply to a problem. When we say that a spring is perfectly, linearly elastic, we mean that Hooke's law $\boldsymbol{F}=-k \boldsymbol{x}$ can be applied. If a body is called 'rigid', it means that its motion can be fully described by the equations $\boldsymbol{F}=m \ddot{\boldsymbol{x}}_{\mathrm{c}}$ and $\boldsymbol{M}_{\mathrm{c}}=\boldsymbol{J} \dot{\boldsymbol{\omega}}+\boldsymbol{\omega} \times \boldsymbol{J} \boldsymbol{\omega}$, with constant $m$ and $\boldsymbol{J}$. When we speak of an ohmic resistor, we mean that the equation $\Delta V=r I$ applies with a constant resistance $r$. Now, when in thermodynamics we say that a process is 'quasi-static' or that the system 'passes through a sequence of equilibrium states', we simply mean that an equation like $p(t) V(t)=M R T(t)$ can be applied at all times. But while the terms 'elastic', 'rigid', and even 'ohmic' give the right ideas, 'quasi-static' is misleading and counter-intuitive: in our exercise we found visible rates of change of $7.5 \mathrm{dm} / \mathrm{s}, 5 \mathrm{kPa} / \mathrm{s}$, and $5 \mathrm{~K} / \mathrm{s}$ (see fig. 4); do we really want to call them 'quasi-static'? Remember that 'static' here means: with reference to the speed of 
sound, $340 \mathrm{~m} / \mathrm{s}$ for air; hence a 'quasi-static' process need not be static at all in the common sense of the word. As for saying that a visibly changing system is 'passing through a sequence of equilibrium states', it sounds to me like saying that a moving object is 'passing through a sequence of states of rest': it is at best an unnecessary and ugly phraseology, at worst simply nonsense. Any mention of 'equilibrium' is misleading when thermodynamic quantities are clearly, visibly changing. Let us keep that term, like 'rest', for situations in which quantities are not changing in time. Instead of mentioning 'instantaneous equilibrium' or 'quasi-static' only to mean that the ideal-gas equation, e.g., can be used at all times, let us say that the system is homogeneous at all times: this implies that it can be described at all times by a single density value, a single pressure value, a single temperature value, etc., instead of field quantities as in continuum thermomechanics. Note in fact the analogy between a homogeneous body and a rigid body: rigidity and homogeneity both imply that the field quantities describing deformations in the full continuum-thermomechanical treatment can be replaced by a smaller set of mechanical or thermal quantities valid for the whole body.

Second: The argument typically adduced for the use of the term 'quasi-static' is that the system can be considered static - in equilibrium - in comparison to some time scale.Yet, we do not call the Space Shuttle's motion at $7600 \mathrm{~m} / \mathrm{s}$ [39] 'quasi-static' — but perhaps we should, given that the motion is in the province of Galileianrelativistic mechanics where every body has to be almost at rest in comparison with the velocity of light? Considering again the analogy between homogeneous and rigid bodies: No body is perfectly rigid, and the equations for a rigid body make sense only if the forces and torques do not exceed the internal ones that keep the body together. Also, the action of a force on a part of the body will not propagate instantly to the rest of the body: waves will propagate in its interior, and they can be detected at appropriate space-time scales. We usually do not point out all these facts when examining a rigid-body problem. Analogously, no body is perfectly homogeneous, and its equations make sense only when changes happen on a smaller scale than the velocity of sound in the body; these changes propagate from point to point and could be detected at appropriate space-time scales. We should feel the need to point out these facts neither more nor less than we do in the case of the rigid body.

Third: It is true that the construction and use of a thermometer 
or a pressure gauge involves notions like response time and coupling (see e.g. Quinn [40]). But so does the construction of positiongauges, velocity-gauges, and dynamometers. Some thermometers are inappropriate when the temperature changes too rapidly, but the same is true of velocity-gauges too. In mechanics and electromagnetics beginning courses the theory of a physical phenomenon and the complex theory of measurement behind it are usually kept quite separate. Positions, accelerations, forces, charges, currents, fields are supposed to be measurable with some appropriate instrument. The same should be true in teaching thermodynamics; cf. Truesdell [9, Lecture 1, pp. 78-79].

Fourth: In mechanics we enjoy the liberty to consider abstract situations leaving unmentioned how they can be realized, in order to concentrate us upon the equations and the phenomenology these exhibit. Springs exactly obeying Hooke's law for arbitrary values of elastic constant and elongation are supposed to exist for the sake of the mathematical problem. Thus it ought to be in thermodynamics, but this liberty is rarely enjoyed there. In mechanics we can say that a particle is in a harmonic potential, without further question about how that is achieved (springs? some kind of field?). Analogously, we should have the right to say, e.g., that the temperature of a system is constant, without needing to mention 'heath baths' all the time. That the temperature is constant, or is changing in some prescribed way, is all we need to know for our problem.

Truesdell makes these points very clear in his typical sharp and witty style; I invite you to read the full book from which this quotation is taken:

'For ten years or more, everyone studying materials has seen that both thermodynamics and mechanics must be brought to bear, but these sciences as hitherto taught do not mesh. Thus, if we take up almost any recent book with "continuum mechanics" or "material science" in its title, we find a chapter on thermodynamics, but that chapter presents a curious contrast with the same author's pages, earlier in the book, on pure mechanics. There the reader is faced by mappings, fields of vectors and tensors, Jacobians, differential invariants, perhaps even Christoffel symbols and affine connections; of course he is presumed familiar with calculus as calculus has been taught for the last fifty years. He can understand dynamical equa- 
tions in tensorial form:

$$
\operatorname{div} \mathbf{T}+\rho \mathbf{b}=\rho \ddot{\mathbf{x}} \quad \text { or } \quad T^{k m}{ }_{, m}+\rho b^{k}=\rho \ddot{x}^{k} .
$$

He is informed, in each case, what the dependent and independent variables are, he is presented with explicit differential equations and boundary-value problems, he is shown many special solutions in concrete cases and is directed to grand tomes where he can find thousands more such solutions not given in the book he is reading, and often he is told about some major problems still unsolved and is challenged to solve them himself. The same reader of the same book then reaches the chapter on thermodynamics, where he is faced with the "axiom"

$$
T d S \geqq \delta Q \text {. }
$$

He is told that $d S$ is a differential, but not of what variables $S$ is a function; that $\delta Q$ is a small quantity not generally a differential; he is expected to believe not only that one differential can be bigger than another, but even that a differential can be bigger than something which is not a differential. He is loaded with an arsenal of words like piston, boiler, condenser, heat bath, reservoir, ideal engine, perfect gas, quasi-static, cyclic, nearly in equilibrium, isolated, universewords indeed familiar in everyday life, doubtless much more familiar than "tangent plane" and "gradient" and "tensor", which he learned to use accurately and fluently in the earlier chapters, but words that never find a place in the mathematical structure at all, words the poor student of science is expected to learn to hurl for the rest of his life in a rhetoric little sharper than a housewife's in the grocery store. The mathematical structure, in turn, is slight. There are no general equations to be solved, no boundary-value or initial-value problems set, no general theorems characterizing classes of solutions. The examples or exercises require no more than calculating partial derivatives or integrals of given functions or their inverses and plugging numbers into the results. The references cited lead to other books containing just the same material, perhaps otherwise explained and ordered, but no broader or clearer in concept, and equally unmathematical. No problems, in the sense that the word "problem" has in the theories of mechanics or electromagnetism or optics or heat conduction, are solved. Neither are any open problems stated. The reader must presume that thermodynamics is an exhausted as well as exhausting subject, with nothing left to be done' [9, Lecture 1, pp. 60-61]. 


\section{Acknowledgements}

Many thanks to Louise, Marianna, Miriam per i loro continui supporto e affetto; to Ingemar Bengtsson and Giacomo Mauro D'Ariano for discussions; to Lucien Hardy and Lee Smolin for encouragement; to swing music; to R. Cheese; and to the developers and maintainers of LTEX, arXiv, Emacs, AUCTEX, MiKTEX, IrfanView, WinDjView, GIMP, Inkscape. Research at the Perimeter Institute is supported by the Government of Canada through Industry Canada and by the Province of Ontario through the Ministry of Research and Innovation.

\section{References}

[1] M. W. Zemansky and R. H. Dittman: Heat and Thermodynamics: An Intermediate Textbook, 7th ed. (New York: McGraw-Hill, 1997).

[2] A. H. Wilson: Thermodynamics and Statistical Mechanics (London: Cambridge University Press, 1957).

[3] H. B. Callen: Thermodynamics and an Introduction to Thermostatistics, 2nd ed. (New York: John Wiley \& Sons, 1985). First publ. 1960.

[4] S. I. Sandler: Chemical, Biochemical, and Engineering Thermodynamics, 4th ed. (New York: John Wiley \& Sons, 2006). First publ. 1977.

[5] M. J. Moran and H. N. Shapiro: Fundamentals of Engineering Thermodynamics, 5th ed. (New York: John Wiley \& Sons, 2006). First publ. 1988.

[6] C. F. Bohren and B. A. Albrecht: Atmospheric Thermodynamics (New York: Oxford University Press, 1998).

[7] D. E. Winterbone: Advanced Thermodynamics for Engineers (London: Arnold, 1997).

[8] C. H. Murphy: Rüchhardt's experiment and atmospheric oscillations. Am. J. Phys. 47/6 (1979), 563-564.

[9] C. A. Truesdell III: Rational Thermodynamics, 2nd ed. (New York: Springer-Verlag, 1984). First publ. 1969.

[10] C. A. Truesdell III and S. Bharatha: The Concepts and Logic of Classical Thermodynamics as a Theory of Heat Engines: Rigorously Constructed upon the Foundation Laid by S. Carnot and F. Reech (New York: SpringerVerlag, 1977).

[11] C. A. Truesdell III: The Tragicomical History of Thermodynamics 18221854 (New York: Springer-Verlag, 1980). 
[12] C. A. Truesdell III: What did Gibbs and Carathéodory leave us about thermodynamics? In: Serrin [41] (1986). Chap. 7, pp. 101-124.

[13] C. Eckart: The thermodynamics of irreversible processes. I. The simple fluid. Phys. Rev. 58/3 (1940), 267-269.

[14] P. W. Bridgman: The Nature of Thermodynamics (Cambridge, USA: Harvard University Press, 1943). First publ. 1941.

[15] K. Menger: The mathematics of elementary thermodynamics. Am. J. Phys. 18/2 (1950), 89-103. See also Thomsen [42]; Menger [43].

[16] C. A. Truesdell III and R. A. Toupin: The Classical Field Theories. In: Flügge [44] (1960), pp. I-VII, 226-858, 859-902. With an appendix on invariants by Jerald LaVerne Ericksen.

[17] C. A. Truesdell III and W. Noll: The Non-Linear Field Theories of Mechanics, 3rd ed. (Berlin: Springer-Verlag, 2004). Ed. by Stuart S. Antman; first publ. 1965.

[18] C. A. Truesdell III: The Elements of Continuum Mechanics (Berlin: Springer-Verlag, 1966).

[19] C. A. Truesdell III: Thermodynamics for beginners. In: Parkus and Sedov [45] (1968), pp. 373-389. With discussion.

[20] D. R. Owen: A First Course in the Mathematical Foundations of Thermodynamics (New York: Springer-Verlag, 1984).

[21] I. Samohýl: Thermodynamics of Irreversible Processes in Fluid Mixtures (Approached by Rational Thermodynamics) (Leipzig: BSB B. G. Teubner Verlagsgesellschaft, 1987).

[22] W. A. Day: A Commentary on Thermodynamics (New York: SpringerVerlag, 1988).

[23] J. L. Ericksen: Introduction to the Thermodynamics of Solids, Revised ed. (New York: Springer, 1998). First publ. 1991.

[24] M. Šilhavý: The Mechanics and Thermodynamics of Continuous Media (Berlin: Springer-Verlag, 1997).

[25] I. Müller: Thermodynamics (Boston: Pitman Advanced Publishing Program, 1985).

[26] J. A. Schufle: Measuring the ratio of specific heats of a gas: Thermodynamic experiments involving harmonic motion. J. Chem. Educ. 34/2 (1957), 7880.

[27] C. Assmann: Ueber Erwärmung und Erkaltung von Gasen durch plötzliche Volumänderung. (Poggendorffs) Ann. d. Phys. u. Chem. 85/1 (1852), $1-36$. 
[28] E. Rüchardt: Eine einfache Methode zur Bistimmung von $c_{p} / c_{v}$. Physik. Zeitschr. 30 (1929), 58-59.

[29] W. L. Burke: Applied Differential Geometry (Cambridge: Cambridge University Press, 1987). First publ. 1985.

[30] W. L. Burke: Div, Grad, Curl Are Dead (1995). http://count.ucsc. edu/ rmont/papers/Burke_DivGradCurl.pdf; 'preliminary draft II'; see also http://www.ucolick.org/ burke/.

[31] A. Bossavit: On the geometry of Electromagnetism (1998). http:// www.lgep.supelec.fr/mocosem/perso/ab/IEEEJapan.html; see also Bossavit [46].

[32] F. W. Hehl and Yu. N. Obukhov: A gentle introduction to the foundations of classical electrodynamics: The meaning of the excitations $(\mathcal{D}, \mathcal{H})$ and the field strengths $(E, B)$ (2000). arXiv: physics/0005084

[33] D. van Dantzig: On the geometrical representation of elementary physical objects and the relations between geometry and physics. Nieuw Archief voor Wiskunde II (1954), 73-89.

[34] D. van Dantzig: The fundamentals equations of electromagnetism, independent of metrical geometry. Proc. Cambridge Philos. Soc. 30/4 (1934), 421-427.

[35] J. A. Schouten: Tensor Analysis for Physicists, Corrected second ed. (New York: Dover Publications, 1989). First publ. 1951.

[36] A. D. Knight and J. R. Levick: Time-dependence of the pressure-volume relationship in the synovial cavity of the rabbit knee. J. Physiol. 335/1 (1983), 139-152.

[37] J. Hardy, A. L. Bertone, and W. W. Muir: Pressure-volume relationships in equine midcarpal joint. J. Appl. Phys. 78/5 (1995), 1977-1984.

[38] A. G. Chynoweth: Dynamic Method for Measuring the Pyroelectric Effect with Special Reference to Barium Titanate. J. Appl. Phys. 27/1 (1956), 7884.

[39] NASA archive (2011). http ://www.nasa.gov/facts/Space/ space_facts_archives.html.

[40] T. J. Quinn: Temperature, 2nd ed. (London: Academic Press, 1990). First publ. 1983.

[41] J. Serrin, ed.: New Perspectives in Thermodynamics (Berlin: SpringerVerlag, 1986).

[42] J. S. Thomsen: [Comment:] The mathematics of elementary thermodynamics. Am. J. Phys. 19/8 (1951), 476. See Menger [15] and also Menger [43].

[43] K. Menger: [Reply:] The mathematics of elementary thermodynamics. Am. J. Phys. 19/8 (1951), 476-477. See Menger [15]; Thomsen [42]. 
[44] S. Flügge, ed.: Handbuch der Physik: Band III/1: Prinzipien der klassischen Mechanik und Feldtheorie [Encyclopedia of Physics: Vol. III/1: Principles of Classical Mechanics and Field Theory] (Berlin: Springer-Verlag, 1960).

[45] H. Parkus and L. I. Sedov, eds.: Irreversible Aspects of Continuum Mechanics and Transfer of Physical Characteristics in Moving Fluids: Symposia Vienna, June 22-28, 1966 (Wien: Springer-Verlag, 1968).

[46] A. Bossavit: Computational Electromagnetism and Geometry (1999-2000). http ://www.lgep.supelec.fr/mocosem/perso/ab/IEEEJapan. html; see also Bossavit [31; 47].

[47] A. Bossavit: On "generalized finite differences": Discretization of electromagnetic problems (2002). http://www.icm.edu.pl/edukacja/mat/ DEP .php; see also Bossavit [31; 46]. 